\title{
SISTEMA PARA EL REGISTRO DE LLAMARADAS SOLARES EN LA BANDA MUY BAJA DE FRECUENCIAS
}

\section{SYSTEM FOR DETECTION OF SOLAR FLARES IN VERY LOW FREQUENCY BAND}

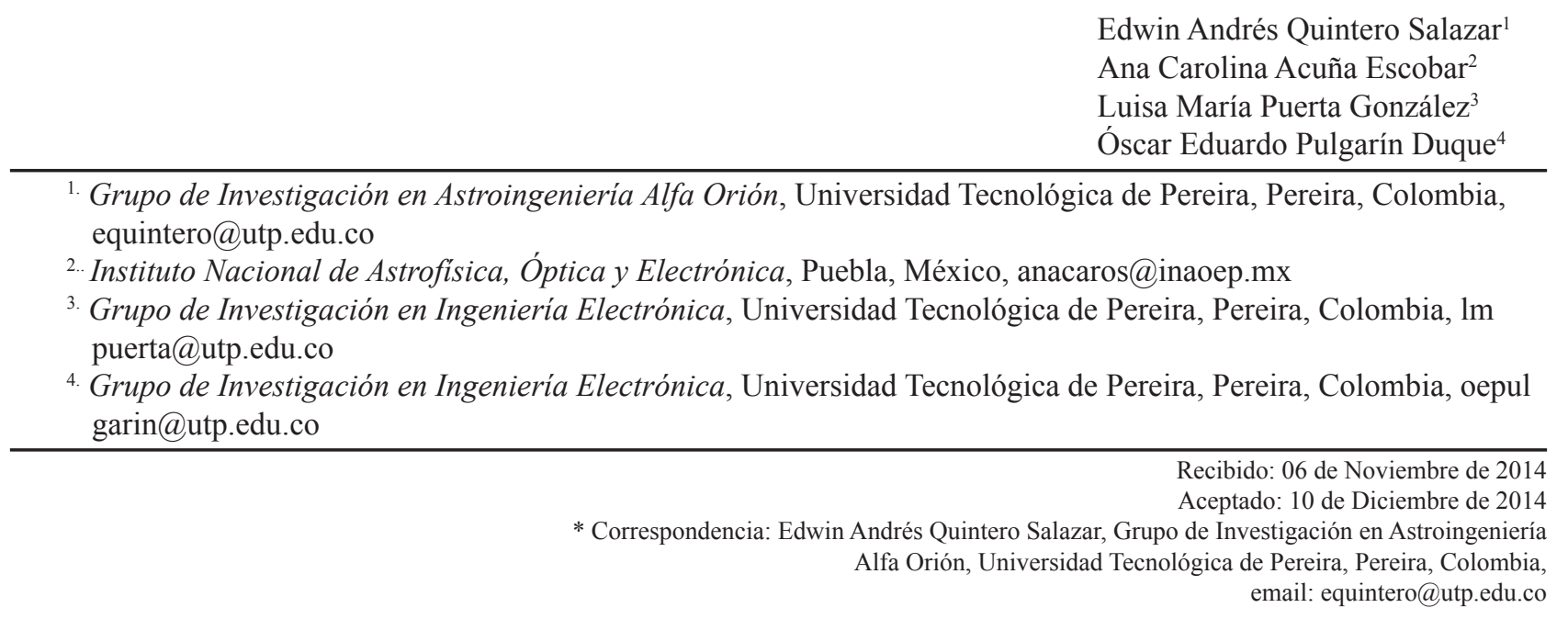

\section{RESUMEN}

En este trabajo se presenta el desarrollo de un sistema para el registro de las actividad solar a partir del monitoreo de las perturbaciones presentadas en las señales de comunicaciones terrestres emitidas en la banda muy baja de frecuencias $(V L F)$. El equipo construido está conformado por una antena tipo lazo, un circuito electrónico para el filtrado y el acondicionamiento de señal, y una interfaz gráfica desarrollada en Matlab ${ }^{\odot}$ para el procesamiento y la visualización de los datos recibidos. El sistema se encuentra instalado actualmente en el Observatorio Astronómico de la Universidad Tecnológica de Pereira, sede del Grupo de Investigación en Astroingeniería Alfa Orión, completando hasta la fecha más de un centenar de erupciones solares detectadas durante 450 días de monitoreo.

Palabras claves: Antena tipo lazo (loop), banda muy baja de frecuencias ( $V L F)$, clima especial, densidad espectral de potencia, fulguración solar

\begin{abstract}
This study presents the development of an electronic system for the registration of solar activity from the monitoring disturbances presented in the terrestrial signals emitted in the very low frequency band. The equipment was built based on a loop type antenna, filtering and amplification circuit for signal conditioning, and a graphical interface developed in Matlab@ for processing and visualization of data collected. The system is currently installed at the Astronomical Observatory of the Technological University of Pereira, home of the Alpha Orion Astroengineering Research Group, completing far more than a hundred solar flares detected over 450 days of monitoring.
\end{abstract}

Keywords: Type antenna loop ( loop), very low frequency band (VLF ), special climate, solar flare, PSD. 


\section{INTRODUCCIÓN}

La importancia actual de las comunicaciones y su vulnerabilidad frente a las alteraciones geomagnéticas producidas por la actividad solar, hacen que el estudio del clima espacial sea de vital importancia alrededor del mundo. Países con grandes infraestructuras invierten en agencias y satélites dedicados solo al estudio del Sol, para así predecir su comportamiento y prevenir grandes apagones y pérdidas en la infraestructura de las comunicaciones. Esto ha conllevado a la creación de diversas redes de trabajo entorno al clima espacial con el fin de monitorear el Sol de una manera más simple desde colegios y universidades situados alrededor del mundo (1), (2), (3), (4), (5), (6), (7).

Los monitores del clima espacial están construidos para registrar los efectos de la actividad solar. Dicha actividad abarca diferentes eventos y fenómenos que no solo se observan y estudian a distancia sino que también afectan la tierra de manera directa. Entre ellos se encuentran las tormentas geomagnéticas provocadas por el viento solar que alteran el campo magnético terrestre, las tormentas de radiación producidas por grandes erupciones solares que aumentan el número de partículas energéticas y el nivel de radiación, los apagones de radio que son producidos por las emisiones de rayos $X$ del Sol (llamaradas tipo $M \circ X$ ) causando perturbaciones en la ionosfera terrestre, y las eyecciones de masa coronal $(C M E)$ que son expulsiones de grandes chorros de plasma que alteran la magnetosfera terrestre (8). Al llegar a la Tierra, gran cantidad de protones provenientes del Sol penetran en las capas altas de la ionosfera, permitiendo a los electrones desprenderse de sus núcleos realizando un proceso de ionización, cambiando la densidad y la localización de sus capas. Con el incremento en la ionización, las señales VLF rebotan en la capa de la ionosfera más cercana a la tierra; de ahí el término "Sudden Ionospheric Disturbance" (SID) que describe los cambios que son monitoreados (9), (10).

Entre las dificultades generadas por el clima espacial se encuentran: fallas en la geo-localización (GPS), las telecomunicaciones, las operaciones vía satélite, el seguimiento espacial, la radionavegación, las redes eléctricas y las redes de transporte de combustible. Esta situación desembocaría en un caos socio-económico que no ha sido previsto debido a que estos fenómenos ocurren de forma intempestiva. Se estima que de presentarse hoy en día una actividad como la del Evento Carrington (1859), los daños ocasionados alcanzarían hasta 20 veces (tres trillones de dólares en el primer año) las pérdidas que fueron provocadas por el huracán Katrina, y tardaría hasta 10 años la recuperación total global (11), (12), (13).

Los monitores de clima espacial son básicamente receptores de ondas VLF. Cuando se registra una fulguración solar dirigida hacia la tierra, las ondas de radio de baja frecuencia transmitidas por centros de comunicación submarina situados en diversos lugares del mundo, son perturbadas por el choque de las llamaradas solares con la ionosfera terrestre, pudiendo ser captadas a lo largo de toda la tierra (9), (10). De esta forma, el presente trabajo se constituye como pionero en la investigación del Sol en el país, ya que plantea el diseño y la construcción del primer sistema para el

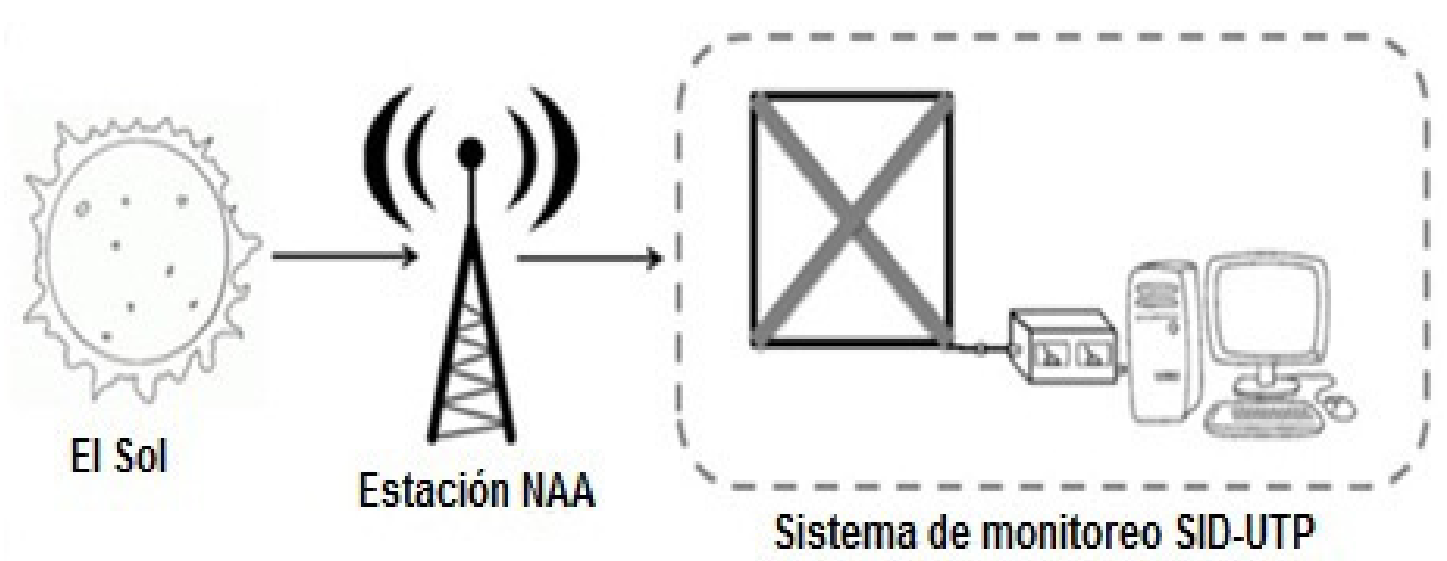

Figura. 1. Diagrama general del monitor SID-UTP. 
monitoreo de la actividad solar en Colombia, involucrando para ello una antena tipo lazo, un circuito de filtrado y amplificación para el acondicionamiento de señal, y una interfaz gráfica para el procesamiento de los datos recopilados.

\section{MATERIALES Y MÉTODOS}

El sistema desarrollado, denominado sistema SIDUTP por sus siglas en inglés (Sudden Ionospheric Disturbance de la Universidad Tecnológica de Pereira), está compuesto de una antena tipo lazo cuadrada para la detección de ondas de baja frecuencia $(V L F)$, un radio receptor que permite filtrar las señales adquiridas específicamente en $24000 \mathrm{~Hz}$, y un equipo de cómputo que recibe la señal filtrada y permite realizar su procesamiento, visualización y almacenamiento para su posterior análisis (14). La Figura. 1 contiene un diagrama general de bloques del equipo desarrollado.

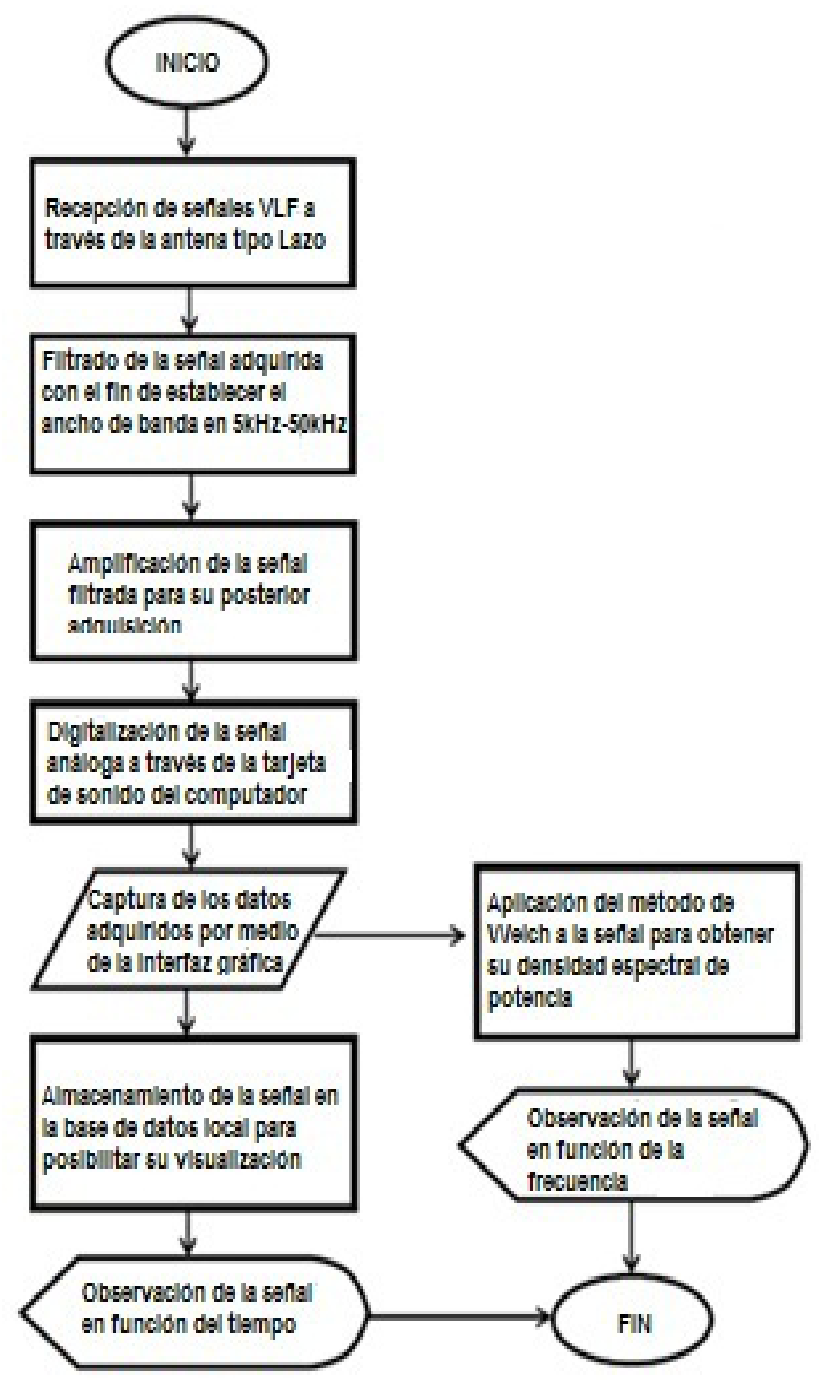

Figura. 2. Funcionamiento del monitor SID-UTP.
La Figura. 2 ilustra la operación del sistema a partir de un diagrama de flujo. Como se observa, las señales recopiladas por la antena son acondicionadas por un sistema de filtrado y enviadas a un computador, quien las recibe a través de su tarjeta de sonido. Una vez allí, las señales capturadas son presentadas tanto en el dominio del tiempo como en el de la frecuencia, este último construido a partir del método de Welch. Las señales procesadas son almacenadas en una base de datos local de tal forma que a partir de su análisis sea posible establecer la existencia y posterior intensidad de las fulguraciones solares presentadas a lo largo del día.

\subsection{ANTENA TIPO LAZO}

Para la adquisición de las señales se construyó una antena tipo lazo de $2 \mathrm{~m}$ de lado con $100 \mathrm{~m}$ de cable aislado calibre $A W G 22$ enrollados sobre una estructura de madera con 25 vueltas, tal y como se muestra en la Figura. 3. Las señales captadas por la antena son transmitidas al radioreceptor a través de un cable coaxial $R G 59$ con malla de ruido, impedancia de $75 \Omega$, y una atenuación de $10 \mathrm{~dB}$ por cada $100 \mathrm{~m}$, generando un nivel cuadrático medio de tensión de $200 \mathrm{mV}$ en el terminal $B N C$ de salida que se encuentra conectado al receptor.

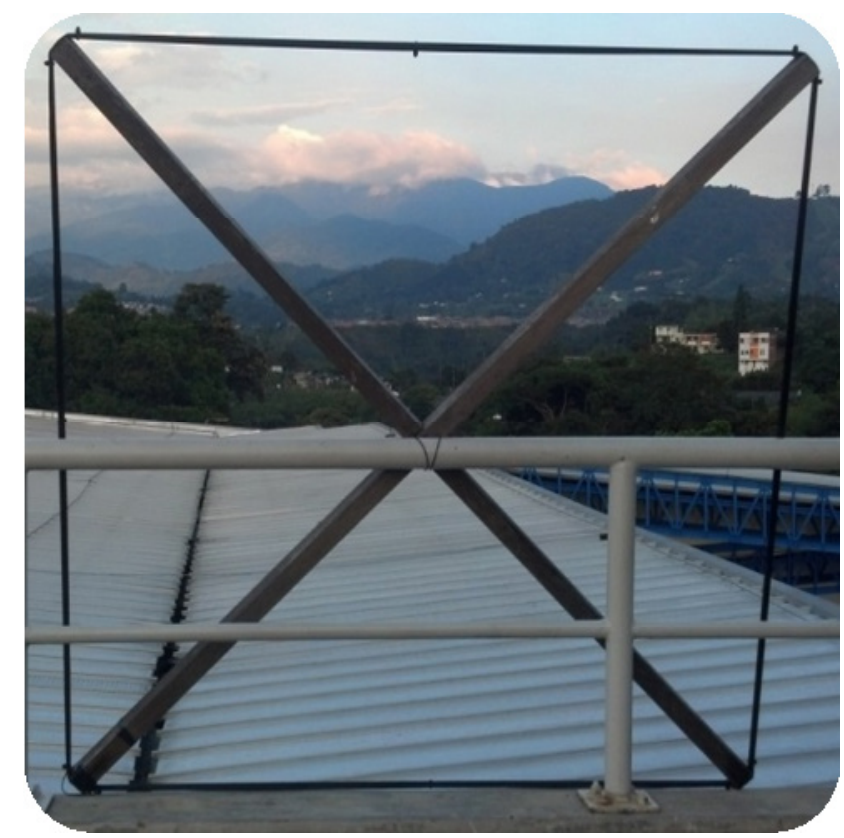

Figura. 3. Antena tipo lazo de $2 m$ del monitor SID-UTP, instalada en el Observatorio Astronómico de la Universidad Tecnológica de Pereira. 


\subsection{RADIORECEPTOR}

Para la recepción de las señales se construyó un circuito de acondicionamiento de señal basado en un filtro activo pasa banda de tercer orden con función de transferencia:

$$
5.984 \times 10^{-7} S
$$

$$
H(s)=\text { - } 1.279 \times 10^{-19} s^{3}+5.123 \times 10^{-14} s^{2}+5.476 \times 10^{-9} s+8.218 \times 10^{-5}
$$

Este filtro fue diseñado con el fin de obtener una ganancia máxima en la banda de frecuencias $5 \mathrm{kHz}-$ $50 \mathrm{kHz}$, que es justamente el intervalo en el que emiten las estaciones de comunicación submarina VLF existentes alrededor del mundo, y que precisamente se ven perturbadas cuando las fulguraciones solares alcanzan la tierra.

En la Figura. 4 es posible observar el diagrama de Bode del filtro construido, obtenida en el entorno de desarrollo integrado Matlab $^{\odot}$ a partir de la función de transferencia, mientras que en las Figura. 5 y 6 se presentan el diagrama esquemático y la respuesta en frecuencia del circuito final construido, respectivamente.

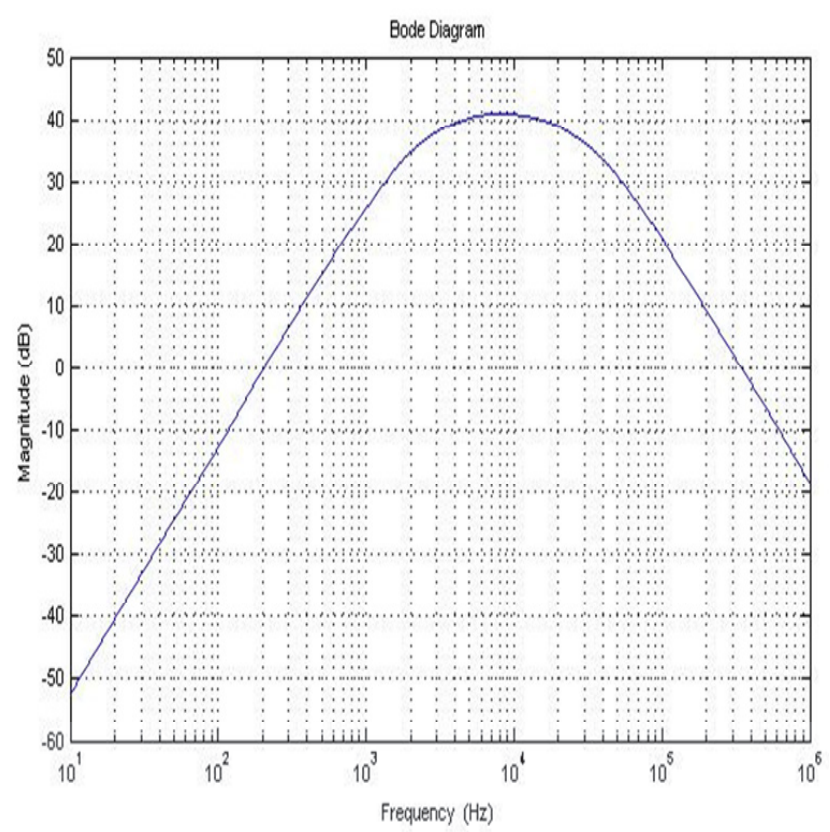

Figura. 4. Diagrama de Bode del filtro diseñado obtenido en Matlab $^{\odot}$.

La señal acondicionada suministrada por el radioreceptor es enviada al computador a través de una tarjeta de sonido configurada a una frecuencia de muestreo de $96000 \mathrm{kHz}$, codificada a 24 bits, de tal forma que pueda digitalizar las señales $V L F$ recibidas.

\subsection{INTERFAZ GRÁFICA}

Para la visualización de las señales capturadas, se desarrolló una interfaz gráfica en Matla $^{\circ}$, que realiza el filtrado digital y la visualización temporal y espectral de las señales provenientes del radio receptor. En la Fig. 7, se observa el diagrama de flujo de la aplicación.

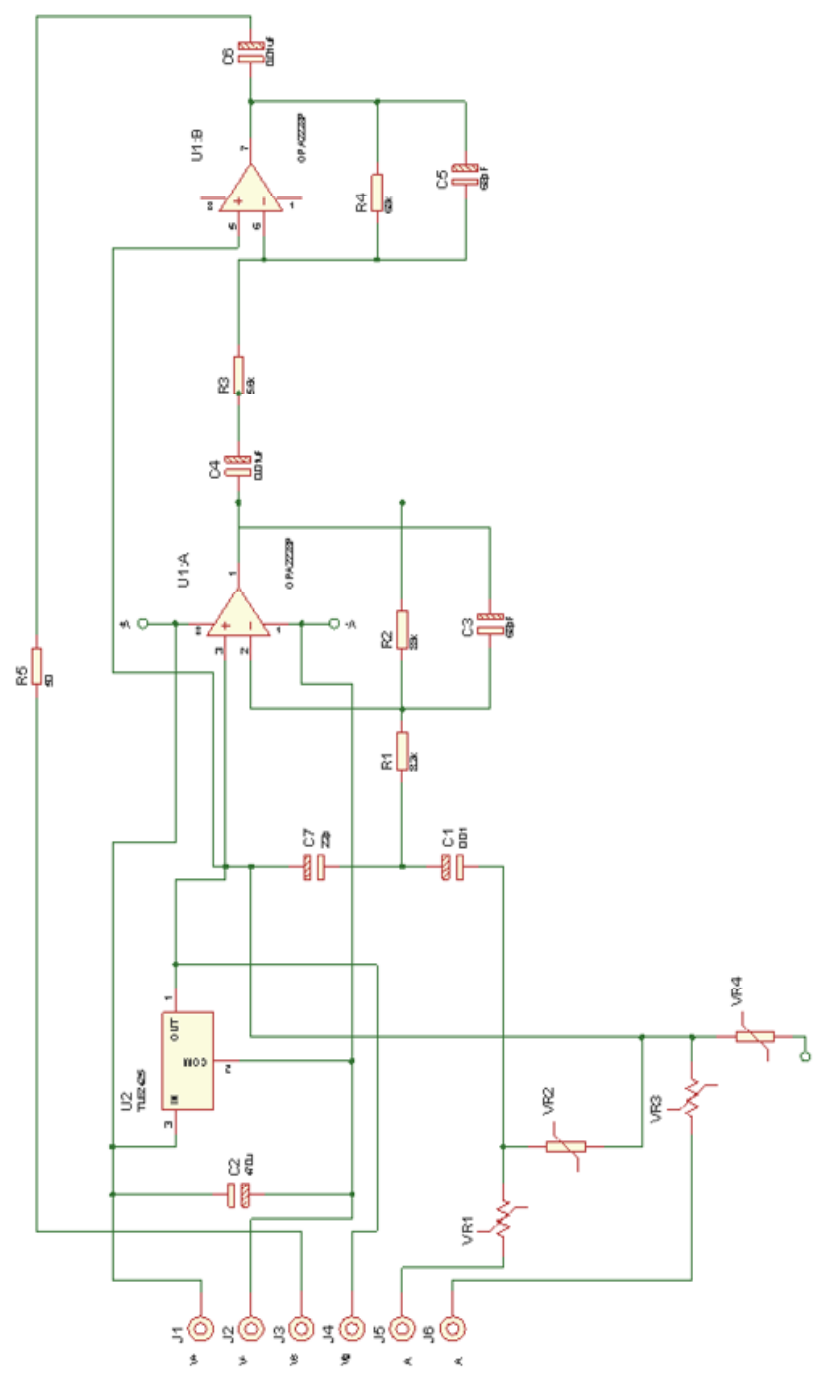

Figura. 5. Diagrama esquemático del filtro pasa banda obtenido en Proteus $^{\odot}$. 


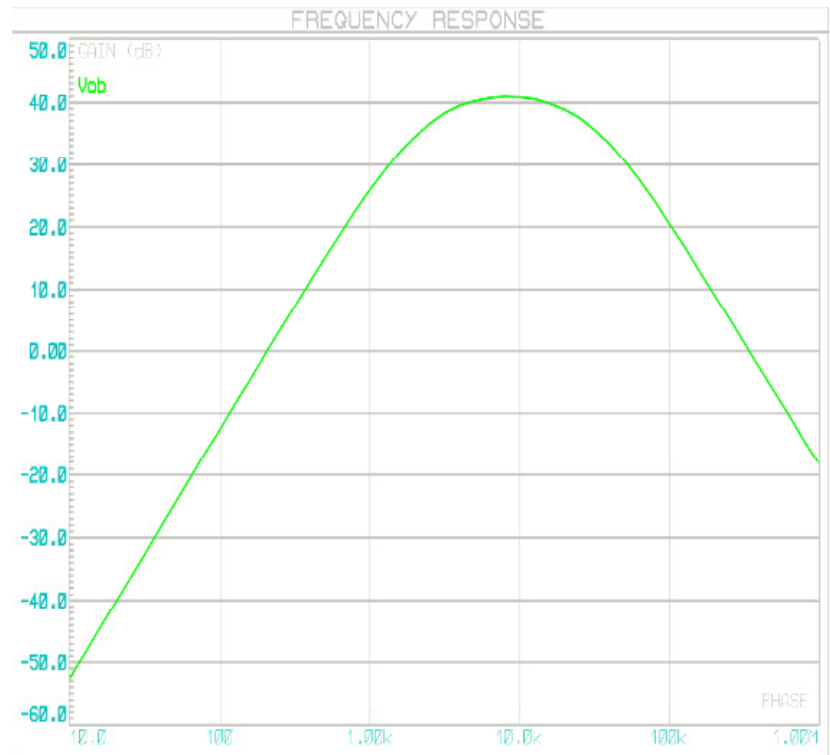

Figura. 6. Respuesta en frecuencia del circuito receptor obtenida en Proteus $^{\mathcal{O}}$.

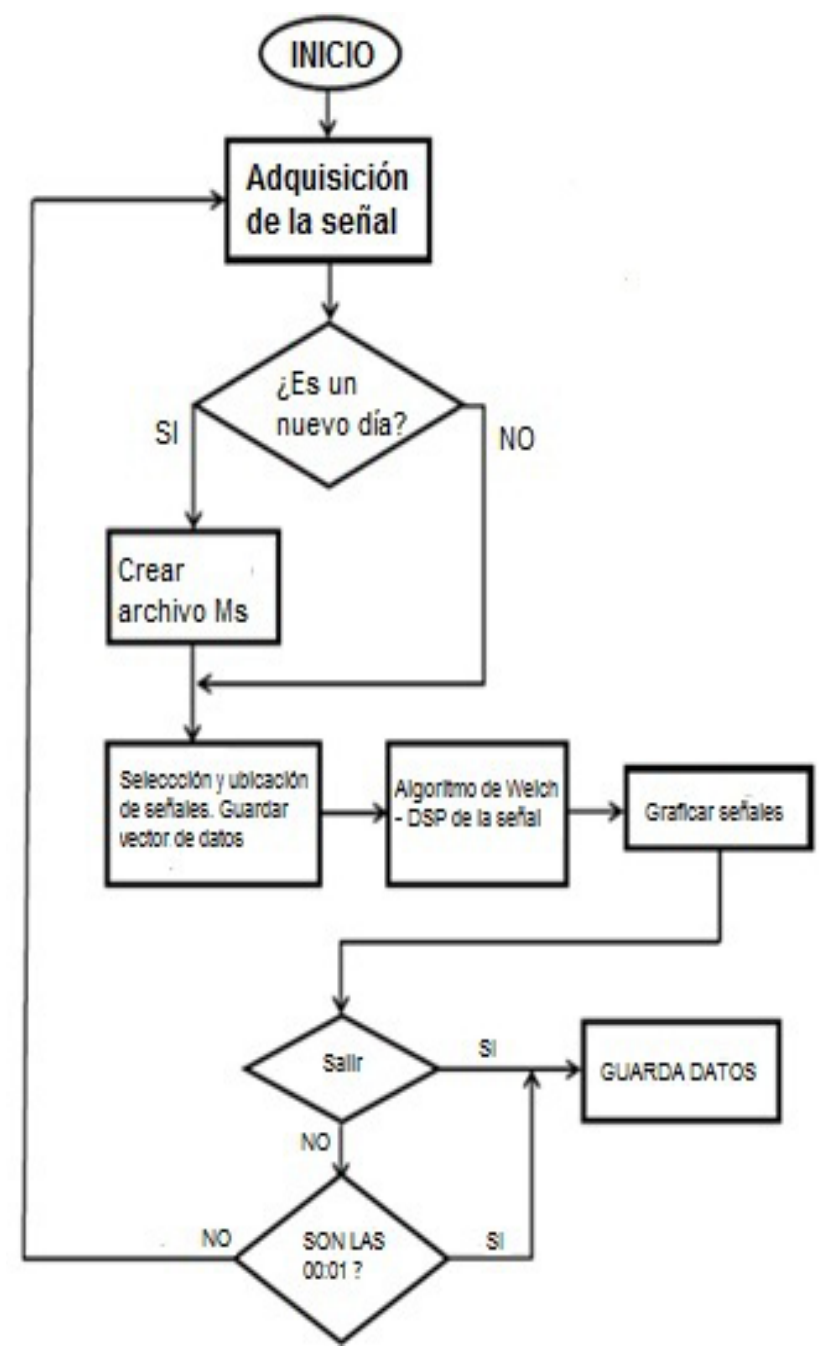

Figura. 7. Diagrana de flujo de la aplicación MonitorCEC.
Para estimar la densidad espectral de potencia de las señales recibidas se utilizó una estrategia no paramétrica basada en la transformada discreta de Fourier que evalúa la señal adquirida en función de la frecuencia, conocida como el método de Welch. Este método promedia periodogramas modificados, los cuales se constituyen como un estimador espectral de la señal que se define a partir de la transformada discreta de Fourier, teniendo en cuenta que en este caso se trata de un proceso estocástico definido como (15):

$$
\mathrm{P}_{\mathrm{k}}=\frac{\left|X_{k}\right|^{2}}{\left(N^{*} T\right)}
$$

Donde:

$X_{\mathrm{k}}$ : Transformada discreta de Fourier de una función de un proceso estocástico $x_{n}$

$T$ : Periodo de muestreo.

El método de Welch divide el registro de $N$ puntos originales en segmentos de $M$ puntos sobrepuestos entre sí y $L$ muestras. Si $L=M$, entonces $N=(K+1) M$, donde $K$ es el número total de segmentos. Posteriormente, el método aplica una ventana a cada segmento para calcular su correspondiente periodograma, de tal forma que el consolidado se consigue promediando todos los periodogramas parciales, obteniendo como resultado el promedio de la densidad espectral de potencia estimada (15).

La Figura. 8 presenta la ventana principal de la aplicación, la cual permite visualizar la señal adquirida, tanto en el dominio del tiempo como en el de la frecuencia. Los datos recopilados de la estación VLF seleccionada son almacenados diariamente en hojas de cálculo, cuyo análisis permite establecer si en el día correspondiente se presentaron eyecciones de masa coronal en el Sol.

\section{RESULTADOS}

El sistema desarrollado inició operaciones el 23 de abril de 2013 desde las instalaciones del Observatorio Astronómico de la Universidad Tecnológica de Pereira, sede del Grupo de Investigación en Astroingeniería Alfa Orión, registrando hasta la fecha más de un centenar de llamaradas solares detectadas. La Fig. 9 presenta una fotografía del sistema instalado y de la aplicación ejecutándose en uno de los equipos de cómputo del observatorio. 


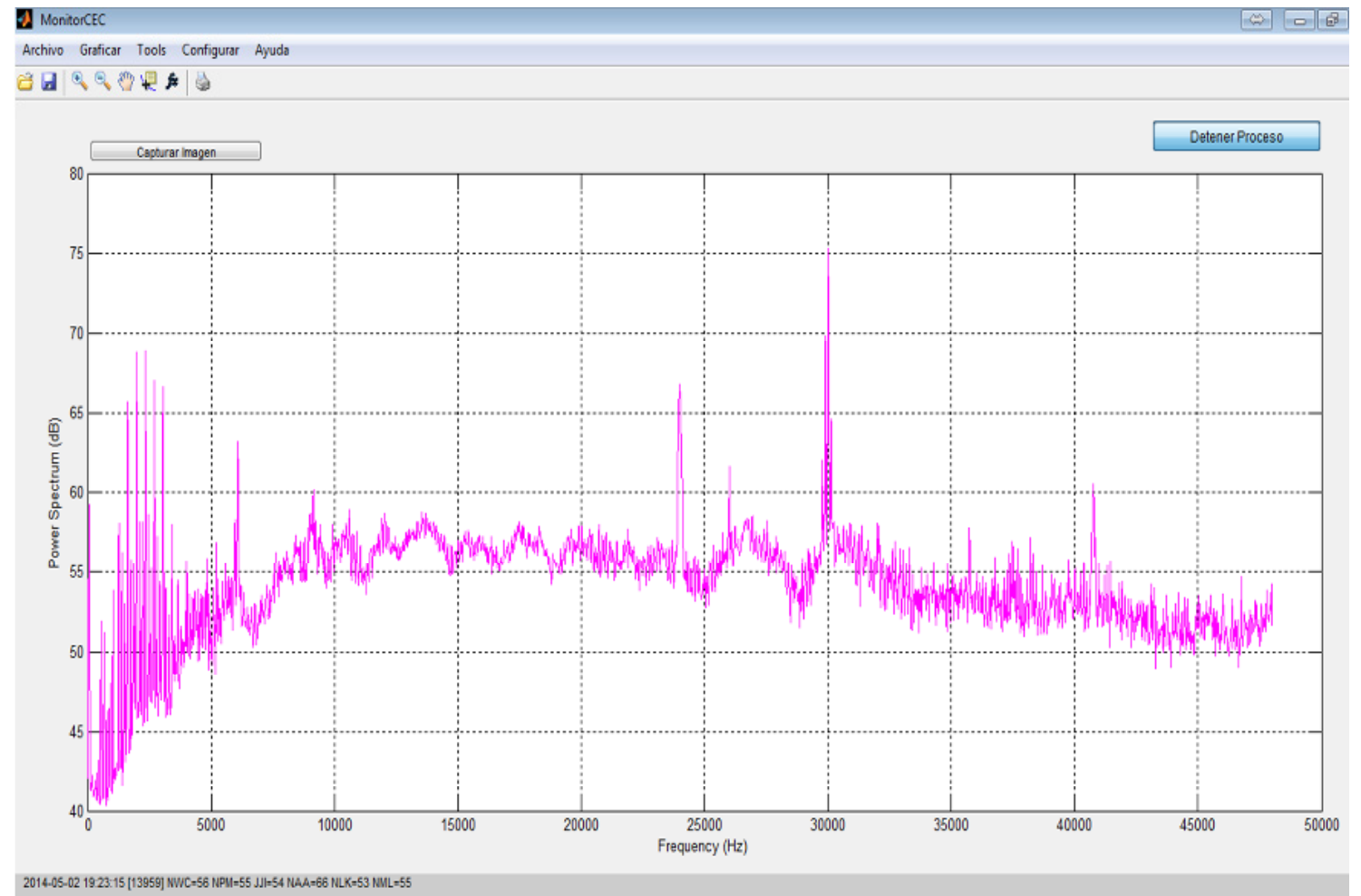

Figura. 8. Ventana principal de la aplicación MonitorCEC.

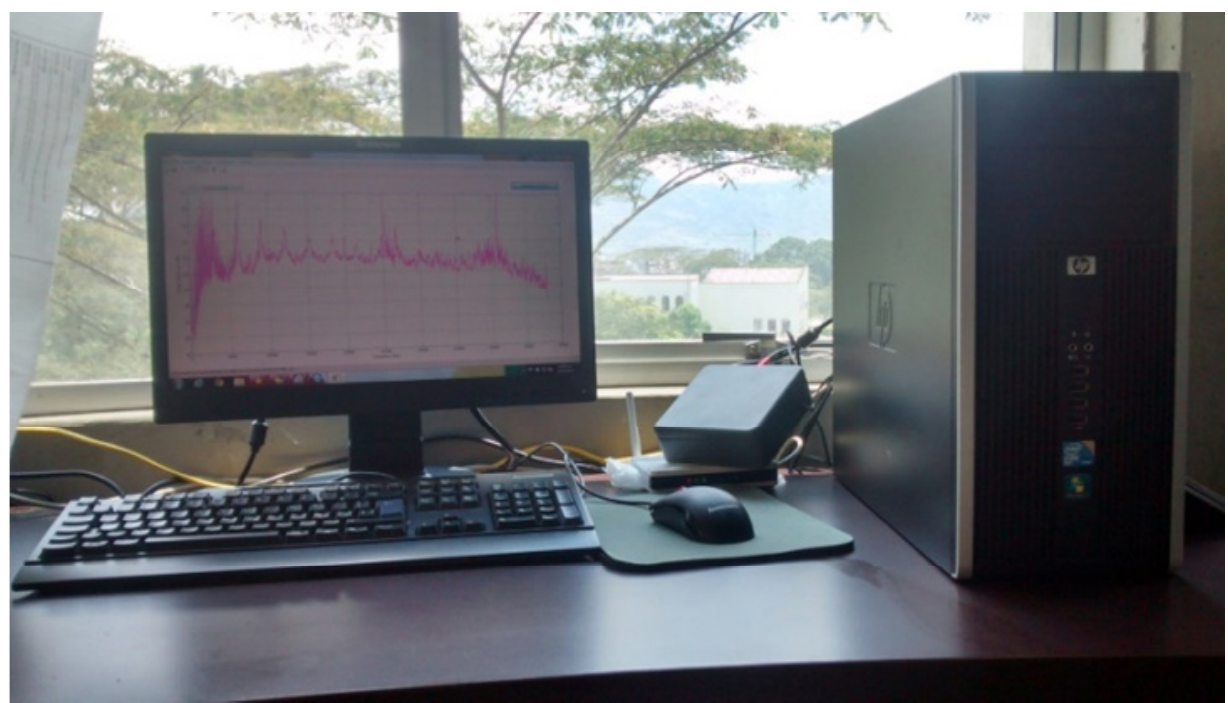

Figura. 9. Fotografía del sistema para el monitoreo del clima espacial en Colombia instalado en el observatorio de la UTP.

Durante el proceso de calibración del equipo, se encontró que al orientar la antena de manera apropiada, la señal $V L F$ que presentaba mejores niveles de relación señal a ruido correspondía a la emitida por la estación NAA desde Cutler, Maine, Estados Unidos, con una potencia de $1000 \mathrm{~kW}$, y en una frecuencia de $24000 \mathrm{kHz}$; armónico que se encuentra resaltado con una elipse de color rojo en el diagrama de densidad espectral de potencia presentado en la Figura. 8.
De acuerdo a su intensidad, las eyecciones de masa coronal se clasifican en los niveles $B, C, M$ y $X$. Solo las fulguraciones catalogadas dentro de las dos últimas categorías poseen la energía suficiente como para generar efectos sobre la actividad terrestre, razón por la cual las detecciones registradas en los resultados pertenecen a estas categorías. Con el fin de validar las detecciones realizadas, las señales son cotejadas con las observaciones del Sol en rayos $X$ realizadas por el satélite $G O E S^{1}$. 
El 29 de marzo de 2014, a las 17:35 GMT, el sistema desarrollado detectó la llamarada solar más intensa del presente año, con una magnitud X1.0, causando, de acuerdo al reporte de la $N O A A^{2}$, un breve apagón de radio en altas frecuencias, generando de esta forma interferencias en sistemas como el radar, GPS, y las comunicaciones por satélite. La Figura. 10 presenta este evento identificado por la línea punteada en color verde. Entre tanto, la Figura. 11 muestra la explosión causante de la emisión de rayos $X$, observada en la banda de los $33.5 \mathrm{~nm}$, originada en gran parte por el Hierro VI, y reportada por el Observatorio de Dinámica Solar $(S D O)$ de la NASA.

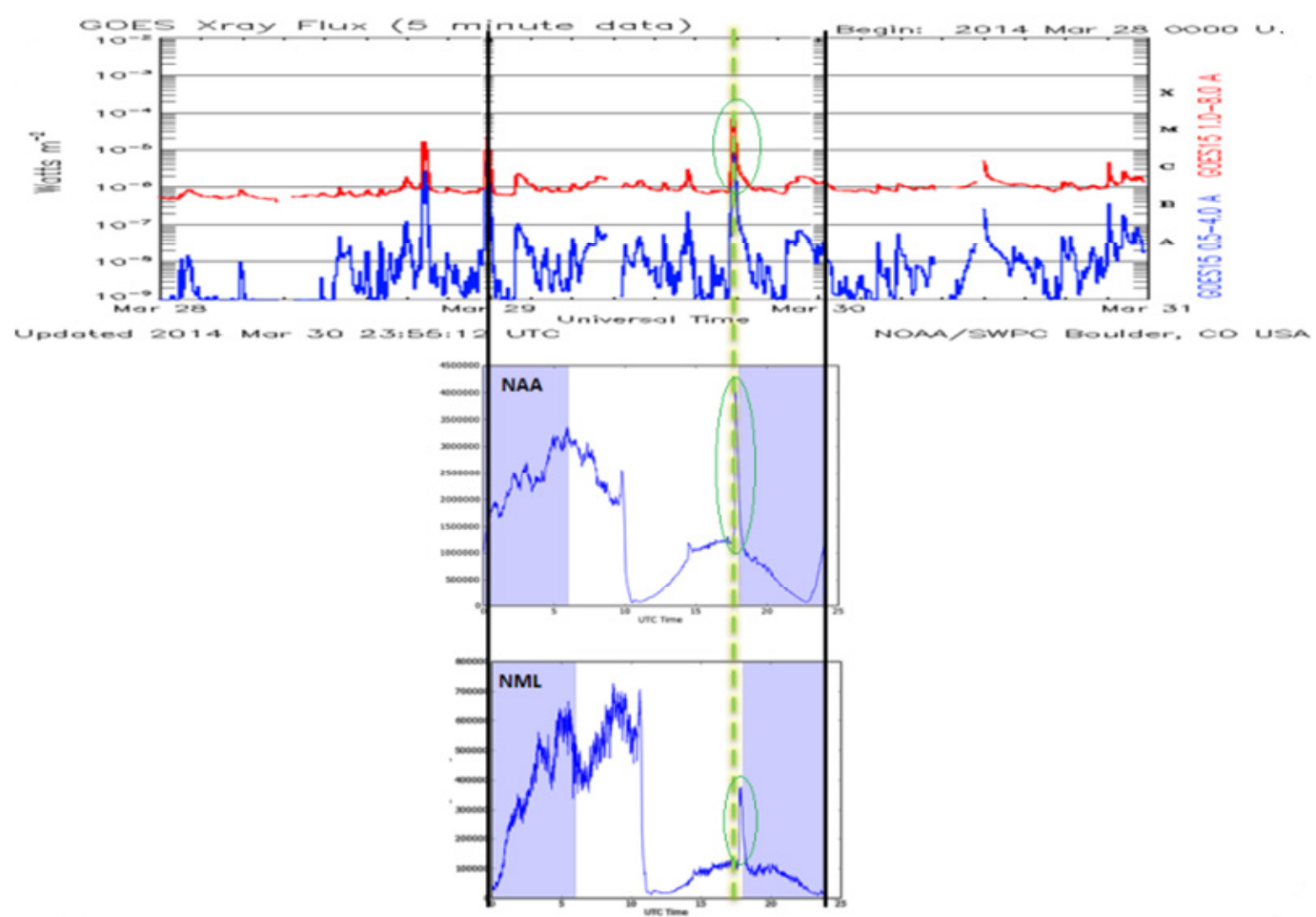

Figura. 10. Llamara de magnitud X1.0 detectada el 29 de marzo de 2014 a las 17:35 GMT (línea punteada en color verde). Arriba: Satélite GOES. Abajo: Monitor SID-UTP.

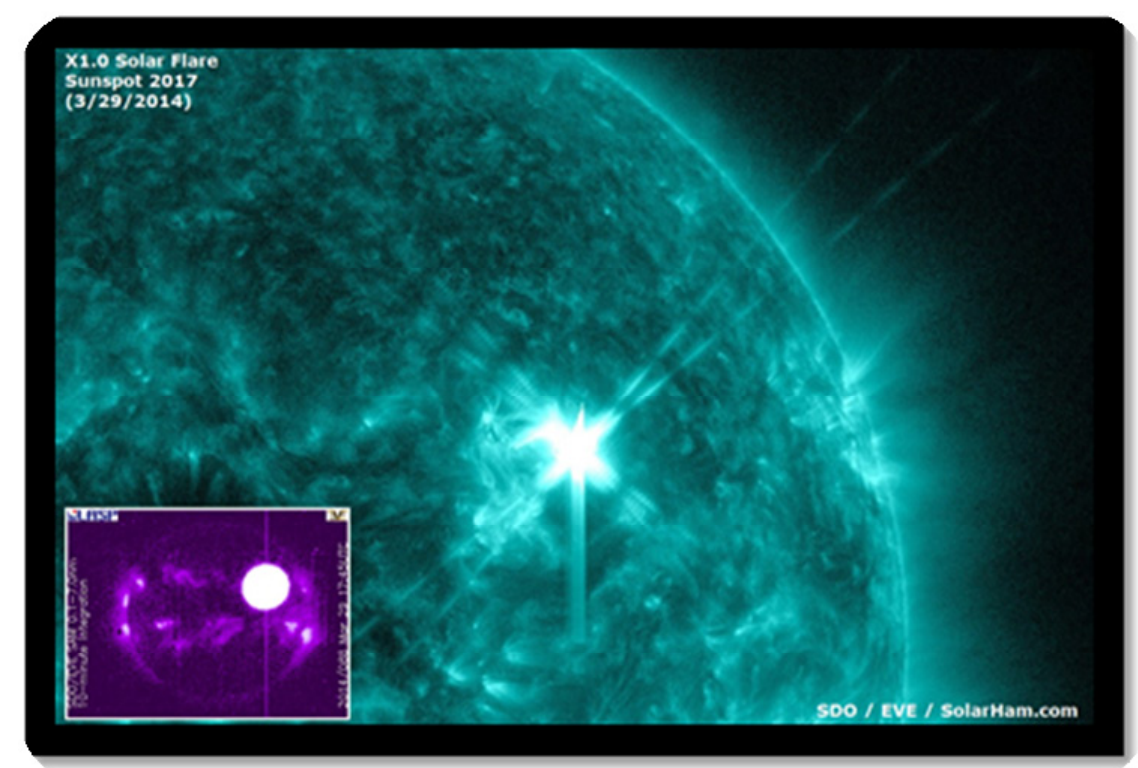

Figura. 11. Explosión solar causante de la llamarada clase $X 1.0$ registrada el 29 de marzo de 2014. Créditos: SDO/EVE/NASA. 


\section{DISCUSIÓN}

Las detecciones de llamaradas solares realizadas por el sistema desarrollado permitieron posicionar al monitor SID-UTP como uno de los equipos pioneros en el monitoreo del clima espacial en Colombia, a tal punto que fue incluido en la red mundial de observatorios de la actividad solar del Stanford Solar Center de la Universidad de Stanford, con el código UTP0383. Por otra parte, y a raíz de la gran cantidad de datos recopilados hasta la fecha, como trabajo futuro se propone el análisis estocástico de los eventos registrados por el sistema para de esta manera construir modelos de predicción que permitan generar alarmas tempranas con el fin de evitar daños en los sistemas de comunicación y en las redes de transporte de energía alrededor del mundo.

Finalmente, vale la pena destacar que debido a que las señales detectadas por el receptor se encuentran dentro de la banda muy baja de frecuencias $(V L F)$, la recepción de las mismas se ve altamente afectada por las tormentas eléctricas, el trazado de la red eléctrica, y la presencia de equipos eléctricos como monitores, televisores, $C P U^{\prime} s$, entre otros. Tal situación exige el cumplimiento de una serie de exigencias a la hora de la instalación de la antena receptora y del trazado de la línea de transmisión coaxial que comunica la antena con el receptor. Para el caso del monitor SID-UTP, la antena fue situada en el extremo occidental de la terraza que rodea la cúpula del observatorio, lejos de los pararrayos del edificio, mientras que la línea de transmisión fue trazada a una distancia prudente con respecto a los conductos que transportan la red eléctrica.

\section{AGRADECIMIENTOS}

Los autores desean expresar sus más sinceros agradecimientos a los miembros del Grupo de Investigación en Astroingeniería Alfa Orión de la Universidad Tecnológica, por apoyar activamente el desarrollo del proyecto, y por facilitar los equipos astronómicos y las instalaciones del observatorio para la instalación y puesta a punto del sistema construido.

\section{BIBLIOGRAFIA}

1. Davies K, Hartmann GK. Studying the Ionosphere with the Global Positioning System. Radio Science Journal. 1997; 32 (4): 1695-9.

2. Zhang DH, Xiao Z. Study of Ionospheric Response to the 4B Flare on 28 October 2003 using International GPS Service Network Data. Journal of Geophysical Research: Space Physics. 2005; 110 (3): 1-7.

3. Rockenbach M, Dal Lago A, Schuch N, Munakata K, Kuwabara T, et al. Global Muon Detector Network used for Space Weather Applications. Space Science Reviews. 2014; 181 (1): 225-10.

4. Tokumitsu M, Ishida Y. A Space Weather Forecasting System with Multiple Satellites based on a Self-recognizing Network. Sensors Journal. 2014; 14 (5): 7974-15.

5. Chakrabarti K, Acharya K, Bose B, Mandal S, Chatterjee A, et al. Monitoring of Sudden Ionospheric Disturbances (SID) from Kolkata (INDIA). Indian Journal of Physics. 2003; 77 (B): 173-4.

6. Raulin J, Correia P, Hadano R, Saraiva A, Correia E, Kaufmann P. The South America VLF NETwork (SAVNET): Development, Installation Status, First Results. Geofísica Internacional. 2009; 48 (3): 253-9.

7. Petrya A, Rodrigues J, Fraga H, Pereira A. Baileye G. First Results of Operational Ionospheric Dynamics Prediction for the Brazilian Space Weather Program. Advances in Space Research. 2014; 54 (1): 22-13.

8. National Oceanic and Atmosferic Admisnistration [Internet], New York: Space Weather Prediction Center; 2005 [consulta el 30 de julio de 2013]. Disponible en: http://www.swpc.noaa. 
gov/NOAAscales/

9. Scherrer D, Clark W, Huynh T, Mitchell R, Roche A, Winegarden S. [Internet], California: SuperSID Manual Stanford University Solar Center; 2010 [consulta el 24 de agosto de 2013]. Disponible en: http://solar-center.stanford.edu/SID/Distribution/SuperSID/supersid_v1_1/ Doc/SuperSIDManual_v1.pdf

10. Scherrer D. [Internet], California: Research with Space Weather Monitor Data Standford Solar Center; 2007 [consulta el 13 de septiembre de 2013]. Disponible en: http://solar-center. stanford.edu/SID/educators/ForTeachers.pdf

11. National Research Council. Severe Space Weather Events--Understanding Societal and Economic Impacts: A Workshop Report. Washington, DC: The National Academies Press, 2008.

12. Tretkoff E. Preparing for the Upcoming Solar Maximum. Space Weather Quarterly. 2009; 7(7): 1-7.

13. National Oceanic and Atmosferic Admisnistration [Internet], New York: Solar Cycle Progression and Prediction; 2009 [consulta el 15 de septiembre de 2013]. Disponible en: http://www. swpc.noaa.gov/SolarCycle/

14. Troshichev O, Janzhura A. Space Weather Monitoring by Ground-Based Means. $1^{\circ}$ Ed. Chichester: Springer Verlag \& Praxis Publishing; 2012.

15. Stoica P, Moses R. Spectral Analysis of Signals. $1^{\circ}$ Ed. New Jersey: Prentice Hall; 2005. 